\title{
Bootstrap Bandwidth Selection Using an $h$-Dependent Pilot Bandwidth
}

\author{
José E. Chacón \\ Departamento de Matemáticas. Avda. de Elvas, s/n. Universidad de \\ Extremadura. 06071 Spain.
}

Keywords: bootstrap bandwidth choice, $h$-dependent pilot bandwidth, kernel density estimation, plug-in method

AMS: $62 \mathrm{G} 07,62 \mathrm{G} 20$

\begin{abstract}
The problem of choosing the bandwidth $h$ for kernel density estimation is considered (see Wand and Jones, 1995). All the plug-in and bootstrap bandwidth selection methods require the use of a pilot bandwidth $g$ and the usual way to make an $h$-dependent choice of $g$ is by obtaining their asymptotic expressions separately and solving the two equations for the sample size $n$. In contrast, we obtain the asymptotically optimal value of $g$ for every fixed $h$, thus making our selection "less asymptotic". Exact error expressions show that some usually assumed hypotheses have to be discarded in the asymptotic study in this case. Based on this idea, two versions of a new bootstrap-type bandwidth selector are proposed, and their properties are analyzed through theoretical results and a simulation study. The new selector can achieve the best possible rate with the minimal asymptotic variance, and the simulations show that in practice one version of this selector provides a good compromise between cross-validation and the popular Sheather-Jones method (Sheather and Jones, 1991): it is not so variable as cross-validation and its centerpoint is significantly less biased than that of the Sheather-Jones bandwidth selector in most cases.
\end{abstract}

\section{Introduction}

One of the main problems in kernel density estimation is the choice of an appropriate bandwidth, as it is well known that the performance of the estimator depends heavily on this parameter. Essentially, there are three possible approaches to automatic bandwidth selection problems: the reference distribution approach, the use of cross-validation techniques and the plug-in ideas.

In many plug-in methods, the pilot bandwidth $g$ is allowed to depend on the original bandwidth $h$. This is done, for instance, in Sheather and Jones (1991). To this end, asymptotic expressions for $h$ and $g$ are given separately and then they are combined to get the dependence between $h$ and $g$, usually following a pattern like $g=C n^{p} h^{m}$ for constants $C, p$ and $m$, where $n$ is the sample size (see also Jones, Marron and Park 1991). However, some of our exact (i.e., non-asymptotic) calculations below show that this kind of parametrization may not be adequate in some cases. In contrast, our goal is to obtain an 
expression for the optimal $g$ as a function of $h$ for every fixed $h$, making the choice "less asymptotic". Then, we provide data-dependent pilot bandwidth selectors aiming to estimate this optimal pilot bandwidth function, and the finite sample performance of the resulting selectors of the original bandwidth is investigated via a simulation study.

\section{Plug-in bandwidth selection}

The kernel density estimator based on a sample $X_{1}, \ldots, X_{n}$ with common density $f$ is given by

$$
f_{n, K, h}(x)=\frac{1}{n} \sum_{i=1}^{n} K_{h}\left(x-X_{i}\right)
$$

where the kernel $K$ is an integrable symmetric function with $\int K=1$, the bandwidth $h$ is a positive real number and we have used the notation $K_{h}(x)=$ $K(x / h) / h$. We will measure the error of this estimator through the mean integrated squared error (MISE), defined by

$$
\operatorname{MISE}(h)=\mathbb{E} \int\left[f_{n, K, h}(x)-f(x)\right]^{2} d x
$$

and our goal is to propose a new bandwidth selector; that is, an estimator of the optimal bandwidth $h_{0 n}=\operatorname{argmin}_{h>0} \operatorname{MISE}(h)$. To this end, we must propose an estimator $\widehat{M}_{n}(h)$ of the MISE function and then define the selector as the minimizer of this criterion function $\widehat{M}_{n}(h)$.

It is easy to show (see Wand and Jones 1995) that the MISE function can be written as $\operatorname{MISE}(h)=R(f)+\frac{R(K)}{n h}+R_{\widetilde{K}, h}(f)$, where we have used the notations

$$
\begin{gathered}
R(L)=\int L^{2}, \quad R_{L, h}(f)=\int\left(L_{h} * f\right) f, \\
\widetilde{K}=\frac{n-1}{n}(K * K)-2 K,
\end{gathered}
$$

with $*$ standing for the convolution operator. Therefore, the optimal bandwidth can be defined also as the minimizer of the function $M(h)=\operatorname{MISE}(h)-R(f)$, which depends on the unknown density $f$ only through the functional $R_{\widetilde{K}, h}(f)$. That is the quantity that we must estimate to provide an estimator of $M(h)$.

Essentially, all plug-in approaches consist of estimating $M(h)$ by smoothing methods and selecting the bandwidth that minimizes the estimated version of $M(h)$. In particular, bootstrap methods use $\hat{M}_{n, L, g}^{*}(h)=R(K) /(n h)+$ $R_{\widetilde{K}, h}\left(f_{n, L, g}\right)$ as an estimate for $M(h)$; see Marron (1992).

The plug-in estimator of the functional $R_{\widetilde{K}, h}(f)$ can be written as

$$
R_{\widetilde{K}, h}\left(f_{n, L, g}\right)=\frac{1}{n^{2}} \sum_{i, j=1}^{n}\left(\widetilde{K}_{h} * \bar{L}_{g}\right)\left(X_{i}-X_{j}\right)
$$


where $\bar{L}=L * L$. It includes the non-random term $\left(\widetilde{K}_{h} * \bar{L}_{g}\right)(0) / n$, so this suggests replacing the bootstrap estimator $\hat{M}_{n, L, g}^{*}$ for its no-diagonal version $M_{n, L, g}^{*}(h)=R(K) /(n h)+T_{n, L, g}(h)$, where

$$
T_{n, L, g}(h)=\frac{1}{n(n-1)} \sum_{i \neq j}\left(\widetilde{K}_{h} * \bar{L}_{g}\right)\left(X_{i}-X_{j}\right) .
$$

Then, we select $H^{*}=\operatorname{argmin}_{h>0} M_{n, L, g}^{*}(h)$.

One of the disadvantages of the plug-in approaches is that they raise the new problem of choosing the pilot bandwidth $g$. Mimicking the steps for selecting $h$, we must evaluate the mean square error (MSE) function of $M_{n, L, g}^{*}(h)$ as an estimate of $M(h), \operatorname{MSE}(h, g)=\mathbb{E}\left[\left\{M_{n, L, g}^{*}(h)-M(h)\right\}^{2}\right]$, and try to estimate the optimal pilot bandwidth, $g_{0 n}(h)$, which minimizes $\operatorname{MSE}(h, g)$ as a function of $g$, i.e., $g_{0 n}(h)=\operatorname{argmin}_{g>0} \operatorname{MSE}(h, g)$. Notice that, using this methodology, the pilot bandwidth must be necessarily local; that is, we must choose one $g$ for every fixed $h>0$.

\section{Exact error formulas}

To provide a new method for choosing the pilot bandwidth we must estimate the MSE of the estimator $M_{n, L, g}^{*}(h)$ in the previous section. As a case-study we have calculated this MSE in the Gaussian case; that is, when all $K, L$ and $f$ correspond to the standard Gaussian density. Once we have an explicit formula for $\operatorname{MSE}(h, g)$ we can apply it to find $g_{0 n}(h)$ numerically in the Gaussian case. We have found that this exact optimal pilot bandwidth exhibits some surprising properties:

1. Figure 1a shows $g_{0 n}(h)$ as a function of $h$ for $n=100$. It is clear that $g_{0 n}(h) \not \rightarrow 0$ as $h \rightarrow 0$; in fact, $g_{0,100}(0) \approx 0.226$. Jones, Marron and Park (1991), generalizing some approaches as Sheather and Jones (1991), suggest taking a factorized pilot bandwidth $g_{n}(h)=C n^{p} h^{m}$ and obtain a remarkably fast convergence rate by choosing appropriate values for $p$ and $m$. Figure 1a shows that in this case this parametrization may not be adequate, in the sense that it would never fulfill the condition that $g_{n}(h)$ be a non-constant function converging to a finite non-zero value as $h \rightarrow 0$.

2. The common assumption when choosing a pilot bandwidth sequence $g_{n}$ is that $h_{0 n} / g_{n} \rightarrow c$ for some $0 \leq c<\infty$ as $n \rightarrow \infty$; see, e.g., Marron (1992). However, in view of Figure 1b we have some evidence that in this case we may expect $h_{0 n} / g_{0 n}\left(h_{0 n}\right) \rightarrow \infty$. 


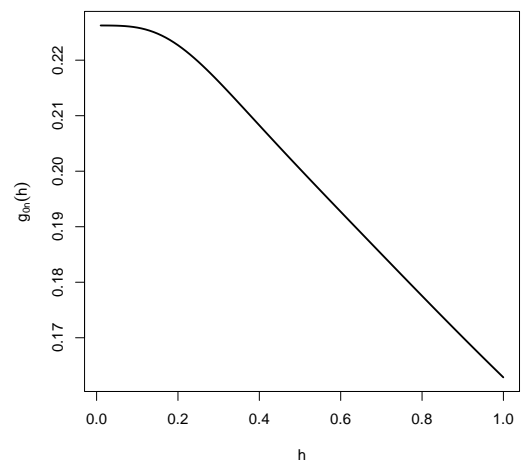

Fig. 1a

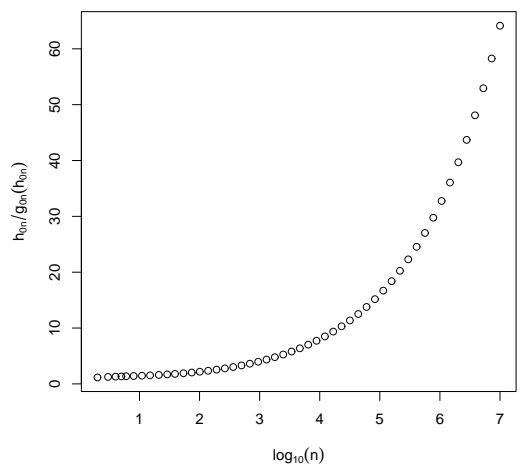

Fig. 1b

\section{Asymptotics}

Let us denote $m_{j}(\alpha)=\int x^{j} \alpha(x) d x / j$ ! and $\left|m_{j}\right|(\alpha)=\int\left|x^{j} \alpha(x)\right| d x / j !$. Then, an integrable function $\alpha$ (not necessarily a kernel) is said to be of finite order if the set $\mathcal{M}_{\alpha}=\left\{j \in \mathbb{N}, j \geq 1:\left|m_{j}\right|(\alpha)<\infty, m_{j}(\alpha) \neq 0\right\}$ is non-empty. In this case, $a=\min \mathcal{M}_{\alpha}$ is called the order of $\alpha$. We will consider here only finite-order symmetric kernel functions. In fact, the following conditions will be assumed:

(K1) $L$ is a symmetric kernel of order $l$ with $\left|m_{l+2}\right|(L)<\infty, K$ is a symmetric kernel of order $k$ with $\left|m_{2 k+2}\right|(K)<\infty$.

(K2) $K$ has $l+2$ bounded continuous derivatives. For $j=0,1, \ldots, l+1$, the $j$ th derivative $K^{(j)}$ is such that $\lim _{|x| \rightarrow \infty} K^{(j)}(x)=0$.

(K3) $L$ has $2 k$ integrable continuous derivatives.

The following result gives an asymptotic expansion of the MSE function with no conditions $f$. The notation $R_{\alpha, \beta, h}(f)=\int\left(\alpha_{h} * f\right)\left(\beta_{h} * f\right) f$ will be used.

Theorem 1. Assume that $K$ and L satisfy conditions (K1) and (K2). Let $g \equiv g_{n}(h)$ be any sequence of bandwidth functions such that $g \rightarrow 0$ pointwise. Then, for any density $f$,

$$
M S E_{n}(h, g)=C_{\sigma 1} / n+C_{\sigma 2} g^{l} / n+C_{\mu}^{2} g^{2 l}+O\left(1 / n^{2}\right)+O\left(g^{l+2} / n\right)+O\left(g^{2 l+2}\right),
$$

for some constants $C_{\sigma 1}, C_{\sigma 2}$ and $C_{\mu}$.

Next we provide asymptotic representations for the optimal pilot bandwidth sequence $g_{0 n}(h)$. For this result we need the following assumption:

(D) The density $f$ has $2 k+l+2$ bounded integrable derivatives. 
Theorem 2. Assume that $K, L$ and $f$ satisfy conditions (K1), (K2) and (D). Then, the optimal pilot bandwidth function $g_{0 n}(h)$ is asymptotically equivalent to

$$
\bar{g}_{0 n}(h)=\left\{\begin{array}{ll}
\left(-C_{\sigma 2} C_{\mu}^{-2} / 2\right)^{1 / l} n^{-1 / l} & \text { if } C_{\sigma 2}<0 \\
0 & \text { if } C_{\sigma 2} \geq 0
\end{array} .\right.
$$

Next we give the asymptotic behavior of the selector $H^{*}$ when $g_{n}(h)$ is any sequence of pilot bandwidth functions mimicking the properties of $g_{0 n}(h)$.

Theorem 3. Suppose that $K, L$ and $f$ satisfy conditions (K1)-(K3) and (D). Let $g_{n}(h)=C_{0}(h) n^{-1 / l}$ be a sequence of positive functions such that

$$
C_{0}\left(h_{0 n}\right) \sim \bar{C}_{0}, \quad C_{0}^{[1]}\left(h_{0 n}\right) / C_{0}\left(h_{0 n}\right) \sim \gamma_{0} h_{0 n}^{2 k-1},
$$

where $\bar{C}_{0}$ and $\gamma_{0}$ are positive constants. Then, the distribution of $n^{r}\left(\frac{H^{*}}{h_{0 n}}-1\right)$ is asymptotically $N\left(0, \sigma^{2}\right)$, where:

i) If $2 \leq l \leq 2 k$, then $r=\frac{1}{4 k+2}$

ii) If $2 k+2 \leq l \leq 4 k$ then $r=\frac{2 l-4 k-1}{2 l}$

iii) If $l \geq 4 k+2$, then $r=\frac{1}{2}$

If $2 \leq l \leq 2 k$ we get the same rate and asymptotic variance as those of the cross-validation method. If $2 k+2 \leq l \leq 4 k$ the rate improves and for $l \geq 4 k+2$ we get the optimal rate $n^{-1 / 2}$ and also the optimal asymptotic variance.

The performance of this new bandwidth selector was evaluated through an extensive simulation study, whose result will be showed in the conference talk.

Acknowledgements: This research has been supported by the Spanish Ministerio de Ciencia y Tecnología project MTM2006-06172.

\section{Bibliography}

[1] Jones, M. C., Marron, J. S. and Park, B. U. (1991). A simple root $n$ bandwidth selector. Ann. Statist., 19, 1919-1932.

[2] Marron, J. S. (1992). Bootstrap bandwidth selection. In Exploring the Limits of Bootstrap, eds. R. LePage and L. Billard, 249-262. Wiley, New York.

[3] Sheather, S. J. \& Jones, M. C. (1991). A reliable data-based bandwidth selection method for kernel density estimation. J. R. Stat. Soc. Ser. B Stat. Methodol., 53, 683-690.

[4] Wand, M. P. \& Jones, M. C. (1995). Kernel smoothing. Chapman \& Hall, London. 